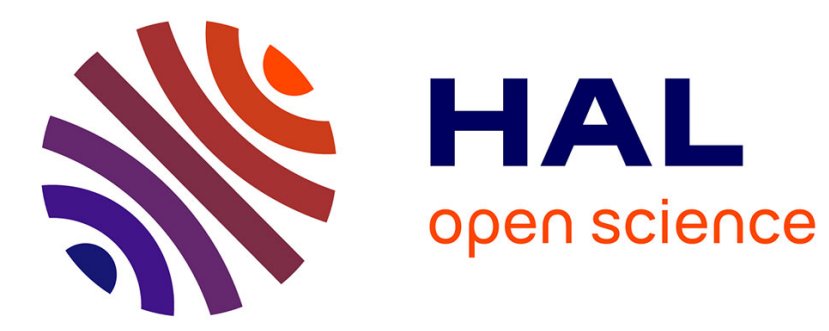

\title{
Hybrid framework for consensus in fleets of non-holonomic robots
}

Tommaso Borzone, Irinel-Constantin Morarescu, Marc Jungers, Michael Boc, Christophe Janneteau

\section{> To cite this version:}

Tommaso Borzone, Irinel-Constantin Morarescu, Marc Jungers, Michael Boc, Christophe Janneteau. Hybrid framework for consensus in fleets of non-holonomic robots. American Control Conference, ACC 2018, Jun 2018, Milwaukee, WI, United States. hal-01756900

\section{HAL Id: hal-01756900 https://hal.science/hal-01756900}

Submitted on 3 Apr 2018

HAL is a multi-disciplinary open access archive for the deposit and dissemination of scientific research documents, whether they are published or not. The documents may come from teaching and research institutions in France or abroad, or from public or private research centers.
L'archive ouverte pluridisciplinaire HAL, est destinée au dépôt et à la diffusion de documents scientifiques de niveau recherche, publiés ou non, émanant des établissements d'enseignement et de recherche français ou étrangers, des laboratoires publics ou privés. 


\title{
Hybrid framework for consensus in fleets of non-holonomic robots
}

\author{
T. Borzone, I.-C. Morărescu, M. Jungers, M. Boc and C. Janneteau
}

\begin{abstract}
This paper presents and analyses a hybrid strategy for the decentralized control of a fleet of non-holonomic robots having a common goal. Each robot has a smooth continuoustime dynamics but in the presented strategy its reference is piecewise constant. Consequently, the hybrid nature of the overall system is introduced by the sporadic updates of the references of the robots. Between two updates of the reference the robots do not need to communicate since each of them will simply apply a reference tracking control. At some discrete time instants the robots update their references based on their relative position with respect to some time-varying neighbors. Our results provide a sufficient consensus (formation realization) condition in term of a minimum duration between consecutive updates of the references. A numerical example illustrates the theoretical results.
\end{abstract}

\section{INTRODUCTION}

New challenges related to networked control systems arose at the end of the 20th century. Since then, this domain is flourishing, because many current engineering problems require multiple systems with local sensing and actions, which have to collaborate in order to accomplish a global goal [1]. For the purposes of formation realization, the robots are often assumed to be very simple, being modeled as single or double integrators [2], [3], [4], [5], [6]. In reality, most mobile robots have non-holonomic dynamics, which are nontrivial to control, as noticed e.g. by [7], [8].

Control and stabilization of unicycle non-holonomic dynamics received a lot of attention during the past decades (see [9] and the references therein). This is partially due to the fact that Brockett's necessary condition [10] for smooth stabilization is not met for this class of vehicles and therefore, no smooth static stabilizing state-feedback control law exists for this type of dynamics. For this reason both discontinuous control laws [11], [12] and time varying [8] control laws have been found to stabilize the center of rotation and the orientation of a single robot. The trajectory tracking control problem with smooth references has also been considered for non-holonomic dynamics via linearization of the error model [7], [13] or via dynamic feedback linearization [14]. Global exponential tracking of smooth trajectories is also presented in [15].

In this work we present a decentralized control strategy for fleets of non-holonomic robots which have to collaborate in

This work was partially funded by the Région Grand Est and CEA Tech Metz

T. Borzone, I.-C. Morărescu and M. Jungers are with Université de Lorraine, CRAN, UMR 7039 and CNRS, CRAN, UMR 7039, France, (tommaso.borzone, constantin.morarescu, marc.jungers) duniv-lorraine.fr

T. Borzone, M. Boc and C. Janneteau are with CEA LIST (Commissariat 1'Energie Atomique - Communicating Systems Laboratory) (michael.boc, christophe.janneteau) dcea.fr order to accomplish a common goal. The proposed algorithm requires sporadic interactions when the robots sense other robots in their neighborhood and based on their relative position with respect to the neighbors they design a constant reference to track. Once the reference is computed the motion of each robot is completely decoupled by the motion of the other robots in the fleet. Although decentralized, this strategy results in a hybrid closed-loop dynamics due to the jumping (non-smooth) references that have to be tracked.

The main contribution of this paper is related to stability analysis of the overall hybrid dynamics in which nonholonomic robots track non-smooth trajectories. Our results provide a sufficient consensus (formation realization) condition in term of a minimum duration between consecutive updates of the references. It is noteworthy that although, in this preliminary work, the communications between robots are assumed undirected and synchronized, we do not impose a fixed network topology. An extension of these results to the case of asynchronous directed communications will be considered in our future works.

The rest of the paper is organized as follows. Section II provides some preliminaries related to the communication network structure and the non-holonomic dynamics under consideration. The control strategy implemented in this work is presented in Section III. The main results concerning stability analysis of the hybrid closed-loop dynamics is reported in Section IV. The results are numerically illustrated in Section $\mathrm{V}$ before providing some concluding remarks.

\section{A. Notation}

The following standard notation has been used throughout the paper. The symbols $\mathbb{N}, \mathbb{R}$ and $\mathbb{R}_{+}$stand respectively for the set of non-negative integers, the set of real and nonnegative real numbers. Given a vector $x$, we denote by $\|x\|$ its Euclidean norm. The transpose of a matrix $A$ is denoted by $A^{\top}$. The notation $A>0(A \geq 0)$ is used for a matrix with positive entries; so the symbols $>$ and $<$ are used to represent element-wise inequalities. A matrix is said to be idempotent if $A^{n}=A, \forall n \in \mathbb{N}_{>0}$. The $k \times k$ identity matrix is denoted $\mathbf{I}_{k}$ and $x\left(t_{k}^{-}\right)=\lim _{t \rightarrow t_{k}, t \leq t_{k}} x(t)$. The column vector of dimension $n$ with all components equal 1 is denoted $\mathbb{1}_{n}$.

\section{PRELIMINARIES}

In this work we present a hybrid formalism for the decentralized control of a fleet of $N$ non-holonomic robots which have to reach consensus or achieve a given formation. This section provides some preliminaries on the interconnection structure, system dynamics and control strategy leading to the hybrid formalism under consideration. 


\section{A. Network structure}

The robots interact with each other through a undirected network described by the time-varying graph $\mathcal{G}(t)=$ $(\mathcal{V}, \mathcal{E}(t))$, where the vertex-set $\mathcal{V}$ represents the set of robots and the edge set $\mathcal{E}(t) \subset \mathcal{V} \times \mathcal{V}$ collects the interactions between robots at time $t$.

Definition 1: A path of length $p$ in a graph $\overline{\mathcal{G}}=(\overline{\mathcal{V}}, \overline{\mathcal{E}})$ is a union of directed edges $\bigcup_{k=1}^{p}\left(i_{k}, j_{k}\right)$ such that $i_{k+1}=$ $j_{k}, \forall k \in\{1, \ldots, p-1\}$. The node $j$ is connected with node $i$ in $\overline{\mathcal{G}}=(\overline{\mathcal{V}}, \overline{\mathcal{E}})$ if there exists at least a path in $\overline{\mathcal{G}}$ from $i$ to $j$ (i.e. $i_{1}=i$ and $j_{p}=j$ ). A connected graph is such that any of its two distinct elements are connected.

Considering the vertex $i$ is characterized by the state $s_{i} \in$ $\mathbb{R}^{n_{s}}, n_{s} \in \mathbb{N}$, a discrete-time linear consensus algorithm is defined by

$$
s(k+1)=\left(P(k) \otimes \mathbf{I}_{n_{s}}\right) s(k)
$$

where $s(k)=\left(s_{1}(k)^{\top}, \ldots, s_{N}(k)^{\top}\right)^{\top}$ and $P(k) \in \mathbb{R}^{N \times N}$ is a row stochastic matrix associated with the graph $\mathcal{G}(k)=$ $(\mathcal{V}, \mathcal{E}(k))$ i.e.

$$
\left\{\begin{array}{l}
P_{i, j}(k)=0, \text { if }(i, j) \notin \mathcal{E}(k), \\
P_{i, j}(k)>0, \text { if }(i, j) \in \mathcal{E}(k), \\
\sum_{j=1}^{N} P_{i, j}(k)=1, \forall i=1, \cdots, N .
\end{array}\right.
$$

The corresponding individual dynamics of each agent is:

$$
s_{i}(k+1)=s_{i}(k)+\sum_{j \neq i} P_{i, j}(k)\left(s_{j}(k)-s_{i}(k)\right)
$$

Throughout the paper we impose the following assumptions.

Assumption 1: For any $k, h \in \mathbb{N}, k \neq h$, if $\mathcal{G}(k)=\mathcal{G}(h)$, then $P(k)=P(h)$.

Assumption 2 (Connectivity): The union digraph $\mathcal{G}=$ $\bigcup_{k \geq k_{0}} \mathcal{G}\left(t_{k}\right)$ is strongly connected for all $k_{0} \in \mathbb{N}$.

Assumption 3 (Diagonal Dominance): The matrix $P_{i, i}\left(t_{k}\right)>\max \{\alpha, \beta\}$ where $\alpha=\sum_{j=1, j \neq i}^{N} P_{i, j}(k)$ and $\beta=\sum_{i=1, i \neq j}^{N} P_{i, j}(k)$ are respectively the row and the column sum of the elements of $P\left(t_{k}\right)$.

Remark 1: It is noteworthy that Assumption 1 limits to a finite cardinality the set of matrices $P(k)$ that are used in dynamics (1).

Under Assumption 1 and 2 it is well-known ([16]) that the discrete-time updating rule (1) ensures asymptotic consensus (i.e. $\left.\lim _{k \rightarrow \infty} s_{i}(k)=\lim _{k \rightarrow \infty} s_{j}(k)\right)$. The Consensus condition can be reformulated imposing the set

$$
\mathcal{A}_{s}=\left\{s \in \mathbb{R}^{N \cdot n_{s}}: s_{i}=s_{j}, \forall(i, j) \in\{1, \ldots, N\}^{2}\right\}
$$

to be globally asymptotically stable for the dynamics (1). On the other hand, since $P(k)$ is row stochastic one has that $P(k) \mathbb{1}_{N}=\mathbb{1}_{N}$. Thus, $\operatorname{span}\left\{\mathbb{1}_{N}\right\}$ is a $P(k)$-invariant subspace. Consequently we can apply the results expressed in [17] and claim that, for any matrix $U \in \mathbb{R}^{(N-1) \times N}$ with Kernel spanned by $\mathbb{1}_{N}$ the equations

$$
U P(k)=Q(k) U, k \in \mathbb{N}
$$

have a unique solutions $Q(k)$, and moreover that

$$
\sigma(P(k))=1 \cup \sigma(Q(k))
$$

where $\sigma$ denotes the spectrum of a matrix. Using these considerations the stability of the attractor $\mathcal{A}_{s}$ for system (2) can be translated as stability of the origin for the following dynamics

$$
s_{p}(k+1)=\left(Q(k) \otimes \mathbf{I}_{n_{s}}\right) s_{p}(k), k \in \mathbb{N},
$$

where $s_{p}(k)=\left(U \otimes \mathbf{I}_{n_{s}}\right) s(k) \in \mathbb{R}^{(N-1) \cdot n_{s}}$. In the sequel we consider $U$ such that $U U^{\top}=\mathbf{I}_{N-1}$. In this case, let us remark that $\left\|U^{\top}\right\|=1$. Indeed applying the definition of matrix norm $\left\|U^{\top}\right\|=\sup _{\|x\|=1} \frac{\left\|U^{\top} x\right\|}{\|x\|}=\sup _{x^{T} x=1} \frac{x^{\top} U U^{\top} x}{x^{T} x}=1$, for $x \in \mathbb{R}^{N-1}$. Moreover, given any vector $s(k)$ there exists a constant $c(k) \in$ $\mathbb{R}^{n_{s}}$ such that

$$
s(k)=\left(U^{\top} \otimes \mathbf{I}_{n_{s}}\right) s_{p}(k)+\mathbb{1}_{N} \otimes c(k) .
$$

where each scalar component $c_{i}(k)$ solves $\left(\mathbf{I}_{n_{s}}-U^{\top} U\right) s_{i}(k)=$ $c_{i}(k) \mathbb{1}_{n_{s}}$.

\section{B. Non-holonomic dynamics}

In the following let us briefly recall the differential equations characterizing a non-holonomic robot dynamics. We assume that a $2 \mathrm{D}$ reference position, denoted by $r_{i}=$ $\left(r_{x_{i}}, r_{y_{i}}\right)$, is provided to each vehicle. Since the problem under study does not require to obtain a specific final orientation of the robots we also assume that the heading reference, denoted by $r_{\theta_{i}}$ is set to 0 for all robots. The Cartesian coordinates of the center of mass of each vehicle with respect to the fixed inertial frame are denoted using vector $X_{i}=\left(x_{i}, y_{i}\right)$. Then, dynamics of the $i^{\text {th }}$ robot is described by the following differential equations

$$
\begin{aligned}
& \dot{e}_{x_{i}}=v_{i} \cos e_{\theta_{i}}, \\
& \dot{e}_{y_{i}}=v_{i} \sin e_{\theta_{i}}, \\
& \dot{e}_{\theta_{i}}=\omega_{i},
\end{aligned}
$$

where $v_{i}$ is the linear velocity and $\omega_{i}$ is the angular velocity of the mobile robot; $e_{x_{i}}$ and $e_{y_{i}}$ are the Cartesian coordinates of the center of mass of the vehicle with respect to a frame positioned on the reference position $r_{i}$, and $e_{\theta_{i}}$ is the angle between the heading direction and the $x$-axis of this frame. In the following, the part of the state representing the 2D Cartesian error coordinates will be denoted as $\varepsilon_{i}=\left(e_{x_{i}}, e_{y_{i}}\right)$. The error coordinates, the reference coordinates and the fixed frame coordinates are related through the transformation

$$
\varepsilon_{i}=X_{i}-r_{i}
$$

The references of the robots are sporadically updated during the reset instants defined by the sequence

$$
\mathcal{T}=\left\{t_{k}: t_{k} \in \mathbb{R}^{+}, t_{k}<t_{k+1}, \forall k \in \mathbb{N}, \lim _{k \rightarrow \infty} t_{k}=\infty\right\} .
$$

Specifically each robot $r_{i}$ is updated via a consensus based map at every $t_{k}$. The sequence of consecutive reference points is thus used as motion planning for the control of each agents. 


\section{CONTROL STRATEGY}

As stated before, we want that the $N$ non-holonomic mobile robots reach a consensus or realize a given translation invariant formation in the Cartesian plane. These two problems are fundamentally identical. Indeed, following [1], let $\Pi$ be the formation that robots have to achieve i.e. $\Pi=\left\{\left(p_{1}^{\top}, \ldots, p_{N}^{\top}\right)^{\top} \mid p_{i} \in \mathbb{R}^{2}, i=1, \cdots, N\right\} \in \mathbb{R}^{2 \cdot N}$. Any collection of agents reference positions $r_{1}, \cdots, r_{N} \in \mathbb{R}^{2}$ is then considered to satisfy the formation requirements if

$$
r_{i}=p_{i}+\tilde{r}^{*}, \quad \forall i=1, \cdots, N,
$$

for some arbitrary translation $\tilde{r}^{*} \in \mathbb{R}^{2}$, the same for all the robots. It is possible to define $\tilde{r}_{i}$ as the displacement of $r_{i}$ from the target location $p_{i}$, i.e. $\tilde{r}_{i}=r_{i}-p_{i}$. Then, algorithm (2) applied to the displacements $\tilde{r}_{i}$ will ensure consensus with the agreement value denoted $\tilde{r}^{*}$. In other words, we translate the invariant formation realization problem in a consensus problem. Consequently, in the following we will focus only on the rendez-vous or consensus problem associated with the $N$ non-holonomic robots. Applying algorithm (3) to the $r_{i}$ for $t_{k} \in \mathcal{T}$ one obtains

$$
r_{i}\left(t_{k}\right)=r_{i}\left(t_{k}^{-}\right)+\sum_{j \neq i} P_{i, j}\left(t_{k}\right)\left(r_{j}\left(t_{k}^{-}\right)-r_{i}\left(t_{k}^{-}\right)\right) .
$$

The usage of the relative distances between references $r_{j}-r_{i}$ requires a communication between the agents to pass the information about their own reference over the network, whereas equipping the robots with relative distance sensors (e.g range and bearing) would lead to the implementation

$$
r_{i}\left(t_{k}\right)=X_{i}\left(t_{k}^{-}\right)+\sum_{j \neq i} P_{i, j}\left(t_{k}\right)\left(X_{j}\left(t_{k}^{-}\right)-X_{i}\left(t_{k}^{-}\right)\right),
$$

for $t_{k} \in \mathcal{T}$. The modification of (11) into (12) comes, as shown in the sequel, with a supplementary cost of a new convergence analysis involving hybrid dynamics. Precisely, we have to consider an augmented state $\left(e_{x_{i}}, e_{y_{i}}, e_{\theta_{i}}, r_{i}^{\top}\right)^{\top}$ and define the flow dynamics characterizing the behavior of the system between two consecutive updates of the reference as well as the jump dynamics related to the update of the reference.

Between two consecutive updates of the reference, formally for $t \in\left[t_{k}, t_{k+1}\right)$, a point stabilization control algorithm is used to drive each vehicle to its reference point $r_{i}$ while the reference is kept constant. Denoting $e_{i}=\left(e_{x_{i}}, e_{y_{i}}, e_{\theta_{i}}\right)^{\top}$ the state of the vehicle, the dynamic in (7) can be rewritten in the more compact form

$$
\dot{e}_{i}=g\left(e_{i}\right) u_{i}, \quad g\left(e_{i}\right)=\left[\begin{array}{cc}
\cos e_{\theta_{i}} & 0 \\
\sin e_{\theta_{i}} & 0 \\
0 & 1
\end{array}\right], \quad u_{i}=\left[\begin{array}{c}
v_{i} \\
\omega_{i}
\end{array}\right] \text {. }
$$

The point stabilization control considered in this work [12] defines a piecewise smooth control law $u_{i}=\kappa\left(e_{i}\right)$ which exponentially stabilizes the origin of the planning reference frame $e_{i}=0$.

It is noteworthy that the jumps of the reference induce jumps on the error state $e_{i}$ too. This generates a coupling between the two dynamics that prevents studying the systems as two separate processes. Basically, $\forall i \in\{1, \ldots, N\}$ we end up with the following hybrid dynamics (see [18] for an exhaustive presentation of hybrid dynamics):

$$
\begin{aligned}
& \left\{\begin{array}{l}
\dot{e}_{i}(t)=g\left(e_{i}(t)\right) k\left(e_{i}(t)\right) \\
\dot{r}_{i}(t)=0
\end{array} \quad \text { for } t \in \mathbb{R}^{+}-\mathcal{T},\right. \\
& \left\{\begin{aligned}
& r_{i}\left(t_{k}\right)=\sum_{j=1}^{n} P_{i, j}\left(t_{k}\right) \varepsilon_{j}\left(t_{k}^{-}\right)+\sum_{j=1}^{n} P_{i, j}\left(t_{k}\right) r_{j}\left(t_{k}^{-}\right) \text {for } t_{k} \in \mathcal{T} . \\
& \varepsilon_{i}\left(t_{k}\right)=\varepsilon_{i}\left(t_{k}^{-}\right)+r_{i}\left(t_{k}^{-}\right)-r_{i}\left(t_{k}\right) \\
& e_{\theta_{i}}\left(t_{k}\right)=e_{\theta_{i}}\left(t_{k}^{-}\right)
\end{aligned}\right.
\end{aligned}
$$

Remark 2: Note that flow dynamics (14) is completely decentralized meaning that each robot tracks its reference and no interaction with other robots is required. Let us also note that the jump map (15) of one robot requires only information from the neighboring (in the interconnection graph) robots.

\section{Stability ANALYSis}

To describe the behavior of the entire fleet it is worth introducing $r=\left(r_{1}^{\top}, \cdots, r_{N}^{\top}\right)^{\top}$ and $\varepsilon=\left(\varepsilon_{1}^{\top}, \cdots, \varepsilon_{N}^{\top}\right)^{\top}$, with $r, \varepsilon \in \mathbb{R}^{2 \cdot N}$. The aim of this section is to show the global asymptotic stability (GAS) of the set $\mathcal{A}$ defined as

$$
\mathcal{A}=\left\{\varepsilon, r \in \mathbb{R}^{2 \cdot N} \mid \varepsilon=0, r_{i}=r_{j}, \forall(i, j) \in\{1, \ldots, N\}^{2}\right\} .
$$

The first requirement in (16) is equivalent to state that each robot reaches its own reference and the second requirement means that all the references achieve consensus.

\section{A. Analysis of the flow dynamics}

The flow dynamics is related to the local stabilization of the vehicles with respect to their relative origins given by the reference state i.e. $\left(r_{x_{i}}\left(t_{k}\right), r_{y_{i}}\left(t_{k}\right)\right)$. Let us recall here the results in [12] where the authors introduce a map $F$ : $\mathbb{R}^{3} \longmapsto \mathbb{R} \times(-\pi, \pi]$ from the state space $e_{i} \in \mathbb{R}^{3}$ to the two-dimensional state space $z_{i} \in \mathbb{R} \times(-\pi, \pi]$

$$
z_{i}=F\left(e_{i}\right) ; \quad F\left(e_{i}\right)=\left[\begin{array}{c}
a_{i}\left(e_{x_{i}}, e_{y_{i}}\right) \\
\alpha_{i}\left(e_{x_{i}}, e_{y_{i}}, e_{\theta_{i}}\right)
\end{array}\right],
$$

where the state variables $a_{i}$ and $\alpha_{i}$ are formally introduced in [12] and illustrated in Fig. 1. Precisely $a_{i}$ represents the length of the arc of the unique circle passing through the points $\left(r_{x_{i}}\left(t_{k}\right), r_{y_{i}}\left(t_{k}\right)\right),\left(x_{i}, y_{i}\right)$ and centered on the vertical line $x=r_{x_{i}}\left(t_{k}\right)$. On the other hand $\alpha_{i}$ is the angle between the heading direction of the $i^{t h}$ robot and the tangent vector to the arc $a_{i}$. Using these new coordinates one can find a feedback control law $u_{i}=h\left(z_{i}\right)$ for the corresponding stabilization problem

$$
\dot{z}_{i}=\frac{\partial F}{\partial e_{i}} \dot{e}_{i}=b\left(e_{i}\right) u_{i}
$$

In particular the closed loop dynamics in terms of the variables $a_{i}$ and $\alpha_{i}$ results completely decoupled

$$
\begin{aligned}
\dot{a}_{i} & =-\gamma b_{1}\left(e_{i}\right)^{2} a_{i}, \\
\dot{\alpha}_{i} & =-k \alpha_{i},
\end{aligned}
$$

where $k$ and $\gamma$ are gains and $b_{1}\left(e_{i}\right)$ is a parameter that depends on the original state $e_{i}$. Moreover, as shown in [12] one can get the following exponential stability result. 


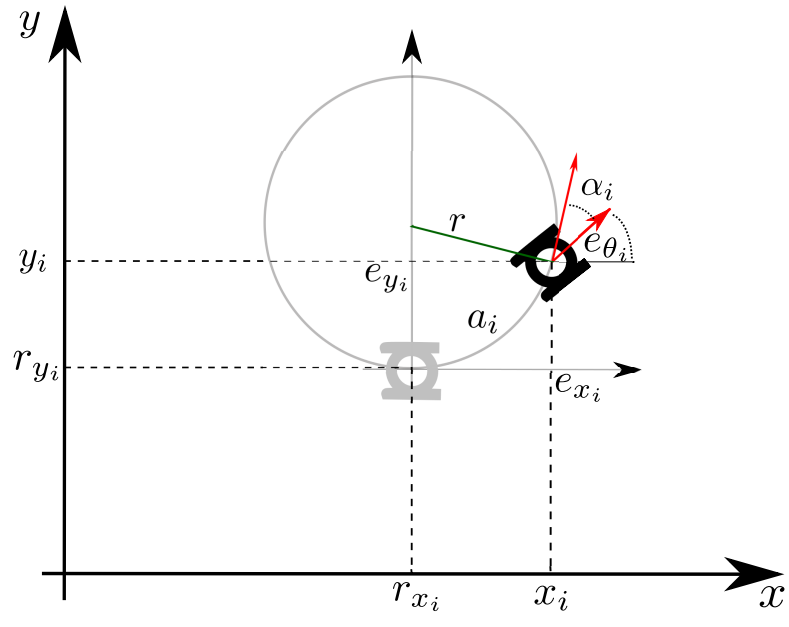

Fig. 1. Scheme showing the state transformation used in [12]. The fixed frame is represented in black while the frame positioned on the vehicle current reference position is represented in grey.

Lemma 1: There exists a control law $h\left(z_{i}\right)$ such that the origin $z_{i}=(0,0)^{\top}$ is globally exponentially stable for the system (18), i.e. there exists some positive constants $c_{z}$ and $\lambda_{z}$ such that

$$
\left\|z_{i}(t)\right\| \leq c_{z}\left\|z_{i}\left(t_{0}\right)\right\| \mathrm{e}^{-\lambda_{z}\left(t-t_{0}\right)}, \text { for } t \geq t_{0} .
$$

Moreover the exponential convergence of $\left\|z_{i}(t)\right\|$ implies the exponential convergence of the trajectories in the space of the errors $e_{i}(t)$ to the origin $e_{i}=0$.

This Lemma leads us to the following instrumental result concerning the behavior of the system (14) over the time interval $\left[t_{k}, t_{k+1}\right)$.

Lemma 2: There exists positive constants $c_{\varepsilon}$ and $\lambda_{\varepsilon}$ such that for $t \in\left[t_{k}, t_{k+1}\right)$

$$
\begin{aligned}
\left\|r_{i}(t)\right\| & =\left\|r_{i}\left(t_{k}\right)\right\| \\
\left\|\varepsilon_{i}(t)\right\| & \leq c_{\varepsilon}\left\|\varepsilon_{i}\left(t_{k}\right)\right\| \mathrm{e}^{-\lambda_{\varepsilon}\left(t-t_{k}\right)}
\end{aligned}
$$

Proof: Due to reasons of space the proof is omitted. Inequality (21) comes directly from the behavior of the reference during the flow (dynamics (14)). As for inequality (22), we claim that expression (20) together with the decoupling which characterizes the closed loop dynamics implies the convergence of the components of $z_{i}$ and in particular

$$
\left|a_{i}(t)\right| \leq c_{a}\left|a_{i}\left(t_{k}\right)\right| \mathrm{e}^{-\lambda_{a}\left(t-t_{k}\right)}, \quad t \in\left[t_{k}, t_{k+1}\right) .
$$

Indeed for $a_{i}\left(t_{k}\right)=0$ the trajectory of $a_{i}$ is identically zero thanks to the decoupling in the dynamics (19), consequently (23) is verified. Moreover, by contradiction, for $a_{i}\left(t_{k}\right) \neq 0$ if the exponential convergence of $a_{i}$ was not respected then neither expression (20) should be true, which contradicts the assumption.

From geometric considerations involving the circle, the arc and the angle at the center we can write $\left\|\varepsilon_{i}\right\| \leq\left|a_{i}\right|$, and $\left|a_{i}\right| \leq \pi / 2\left\|\varepsilon_{i}\right\|$. These two expressions can be used in (23) yielding $\left\|\varepsilon_{i}(t)\right\| \leq c_{a} \frac{\pi}{2}\left\|\varepsilon_{i}\left(t_{k}\right)\right\| \mathrm{e}^{-\lambda_{a}\left(t-t_{k}\right)}, \forall t \in\left[t_{k}, t_{k+1}\right)$.
We can now state the following Corollary that is straightforward from Lemma 2.

Corollary 1: There exists positive constants $c_{\varepsilon}$ and $\lambda_{\varepsilon}$ such that for $t \in\left[t_{k}, t_{k+1}\right)$

$$
\begin{aligned}
&\|r(t)\|=\left\|r\left(t_{k}\right)\right\| \\
&\|\varepsilon(t)\| \leq c_{\varepsilon}\left\|\varepsilon\left(t_{k}\right)\right\| \mathrm{e}^{-\lambda_{\varepsilon}\left(t-t_{k}\right)} .
\end{aligned}
$$

Corollary 1 basically states that, as far as the reference is fixed, one can design a decentralized controller that exponentially stabilizes system (14).

\section{B. Analysis of the jump map}

In the previous subsection, we have shown that for $t \in$ $\left[t_{k}, t_{k+1}\right)$ the Cartesian positioning error of the vehicles converges toward $\varepsilon=\mathbf{0}$ but nothing can be said about the reference $r$ which is kept constant during the flow. In order to achieve the global asymptotic stability of $\mathcal{A}$ defined in (16), let us investigate the behavior of system (15).

First, let us notice that $\mathrm{e}_{\theta_{i}}$ does not change during the jumps defined by (15) and therefore we can neglect this variable in the subsequent analysis. Moreover, by collecting all the dynamics in (15) one obtains the following discretetime system:

$$
\begin{aligned}
& r\left(t_{k}\right)=\mathcal{P}\left(t_{k}\right) r\left(t_{k}^{-}\right)+\mathcal{P}\left(t_{k}\right) \varepsilon\left(t_{k}^{-}\right) \\
& \varepsilon\left(t_{k}\right)=\left(\mathbf{I}_{2 N}-\mathcal{P}\left(t_{k}\right)\right) \varepsilon\left(t_{k}^{-}\right)+\left(\mathbf{I}_{2 N}-\mathcal{P}\left(t_{k}\right)\right) r\left(t_{k}^{-}\right),
\end{aligned}
$$

for $t_{k} \in \mathcal{T}$ and where $\mathcal{P}\left(t_{k}\right)=P\left(t_{k}\right) \otimes \mathbf{I}_{2}$.

Remark 3: The matrices

$$
\overline{\mathcal{P}}\left(t_{k}\right)=\left(\begin{array}{cc}
\mathcal{P}\left(t_{k}\right) & \mathcal{P}\left(t_{k}\right) \\
\mathbf{I}_{2 N}-\mathcal{P}\left(t_{k}\right) & \mathbf{I}_{2 N}-\mathcal{P}\left(t_{k}\right)
\end{array}\right)
$$

are idempotent i.e. $\overline{\mathcal{P}}^{2}\left(t_{k}\right)=\overline{\mathcal{P}}\left(t_{k}\right)$.

In the following, the first equation in (25) is multiplied to the left with $\mathcal{U}=U \otimes \mathbf{I}_{2}$ and taking into account (4) one obtains for $t_{k} \in \mathcal{T}$

$$
\left\{\begin{aligned}
\mathcal{U} r\left(t_{k}\right) & =Q\left(t_{k}\right) \mathcal{U} r\left(t_{k}^{-}\right)+\mathcal{Q}\left(t_{k}\right) \mathcal{U} \varepsilon\left(t_{k}^{-}\right) \\
\varepsilon\left(t_{k}\right) & =\left(\mathbf{I}_{2 N}-\mathcal{P}\left(t_{k}\right)\right) r\left(t_{k}^{-}\right)+\left(\mathbf{I}_{2 N}-\mathcal{P}\left(t_{k}\right)\right) \varepsilon\left(t_{k}^{-}\right),
\end{aligned}\right.
$$

where $Q\left(t_{k}\right)=Q\left(t_{k}\right) \otimes \mathbf{I}_{2}$ with $Q\left(t_{k}\right)$ defined in (4). Let $r_{p}\left(t_{k}\right)=\mathcal{U} r\left(t_{k}\right)$ and note that using (6) one has

$$
\left(\mathbf{I}_{2 N}-\mathcal{P}\left(t_{k}\right)\right) r\left(t_{k}^{-}\right)=\left(\mathbf{I}_{2 N}-\mathcal{P}\left(t_{k}\right)\right) \mathcal{U}^{\top} r_{p}\left(t_{k}^{-}\right) .
$$

Consequently, (26) rewrites for $t_{k} \in \mathcal{T}$ as

$\left\{\begin{aligned} r_{p}\left(t_{k}\right) & =Q\left(t_{k}\right) r_{p}\left(t_{k}^{-}\right)+Q\left(t_{k}\right) \mathcal{U} \varepsilon\left(t_{k}^{-}\right) \\ \varepsilon\left(t_{k}\right) & =\left(\mathbf{I}_{2 N}-\mathcal{P}\left(t_{k}\right)\right) \mathcal{U}^{\top} r_{p}\left(t_{k}^{-}\right)+\left(\mathbf{I}_{2 N}-\mathcal{P}\left(t_{k}\right)\right) \varepsilon\left(t_{k}^{-}\right) .\end{aligned}\right.$

$<$ normalsize Under Assumption 1 the following quantities are well defined (i.e. the maximum exists):

$$
\begin{aligned}
& \gamma_{11}=\max _{t_{k} \in \mathcal{T}}\left\|Q\left(t_{k}\right)\right\|, \\
& \gamma_{12}=\max _{t_{k} \in \mathcal{T}}\left\|Q\left(t_{k}\right) \mathcal{U}\right\|, \\
& \gamma_{21}=\max _{t_{k} \in \mathcal{T}\left\|\left(\mathbf{I}_{2 N}-\mathcal{P}\left(t_{k}\right)\right) \mathcal{U}^{\top}\right\|,} \gamma_{22}=\max _{t_{k} \in \mathcal{T}\left\|\left(\mathbf{I}_{2 N}-\mathcal{P}\left(t_{k}\right)\right)\right\| .}
\end{aligned}
$$

Lemma 3: Under Assumption $1, \forall t_{k} \in \mathcal{T}$, one has that:

$$
\begin{aligned}
\left\|r_{p}\left(t_{k}\right)\right\| & \leq \gamma_{11}\left\|r_{p}\left(t_{k}^{-}\right)\right\|+\gamma_{12}\left\|\varepsilon\left(t_{k}^{-}\right)\right\|, \\
\left\|\varepsilon\left(t_{k}\right)\right\| & \leq \gamma_{21}\left\|r_{p}\left(t_{k}^{-}\right)\right\|+\gamma_{22}\left\|\varepsilon\left(t_{k}^{-}\right)\right\| .
\end{aligned}
$$

Proof: The proof is straightforward from (27) and the definition of $\gamma_{11}, \gamma_{12}, \gamma_{21}$ and $\gamma_{22}$ in (28). 


\section{Overall hybrid system stability analysis}

In the sequel we consider the matrices $\Gamma=\left(\begin{array}{ll}\gamma_{11} & \gamma_{12} \\ \gamma_{21} & \gamma_{22}\end{array}\right)$ and $M_{\tau}=\left(\begin{array}{cc}1 & 0 \\ 0 & c_{\varepsilon} \mathrm{e}^{-\lambda_{\varepsilon} \tau}\end{array}\right)$. We emphasize that the behavior of $\|\varepsilon(t)\|$ and $\|r(t)\|$ (thus $\left.\left\|r_{p}(t)\right\|\right)$ is characterized within $\left[t_{k}, t_{k+1}\right.$ ) by Corollary (1) in term of the matrix $M_{t_{k+1}-t_{k}}$. On the other hand Lemma 3 gives an upper-bound, in term of $\Gamma$, on the jumps that $\|\varepsilon(t)\|$ and $\left\|r_{p}(t)\right\|$ suffer at time $t_{k} \in \mathcal{T}$.

Lemma 4: Under Assumption 1, let $\tau^{*} \geq 0$ such that the positive matrix $\Gamma M_{\tau^{*}}$ is Schur. Then, for all sequences $\mathcal{T}=\left(t_{k}\right)_{k \geq 0}$ of jump times satisfying the dwell-time property $t_{k+1}-t_{k} \geq \tau^{*}$, for all $k \in \mathbb{N}$, $\mathcal{A}$ is GAS with respect to dynamics (14)-(15). $k \in \mathbb{N}$,

Proof: From Corollary 1 and Lemma 3, it follows that for all

$$
\left(\begin{array}{c}
\left\|r_{p}\left(t_{k}\right)\right\| \\
\left\|\varepsilon\left(t_{k}\right)\right\|
\end{array}\right) \leq \Gamma M_{\tau_{k-1}} \ldots \Gamma M_{\tau_{0}}\left(\begin{array}{c}
\left\|r_{p}\left(t_{0}\right)\right\| \\
\left\|\varepsilon\left(t_{0}\right)\right\|
\end{array}\right),
$$

where $\tau_{k}=t_{k+1}-t_{k}, \forall k \in \mathbb{N}$. Remarking that the coefficients of the positive matrix $M_{\tau}$ are non-increasing with respect to $\tau$, it follows that

$$
\left(\begin{array}{c}
\left\|r_{p}\left(t_{k}\right)\right\| \\
\left\|\varepsilon\left(t_{k}\right)\right\|
\end{array}\right) \leq\left(\Gamma M_{\tau^{*}}\right)^{k}\left(\begin{array}{c}
\left\|r_{p}\left(t_{0}\right)\right\| \\
\left\|\varepsilon\left(t_{0}\right)\right\|
\end{array}\right) .
$$

Hence, if the positive matrix $\Gamma M_{\tau^{*}}$ is Schur, then both sequences $\left(\left\|r_{p}\left(t_{k}\right)\right\|\right)_{k \geq 0}$ and $\left(\left\|\varepsilon\left(t_{k}\right)\right\|\right)_{k \geq 0}$ go to 0 , and the system (14)-(15) converges to some point in $\mathcal{A}$.

Hence, the stability of $\mathcal{A}$ with respect to the overall hybrid dynamics of the fleet of robots can be investigated by studying the spectral properties of the positive matrix $\Gamma M_{\tau^{*}}$. Let us remark that values $\tau^{*}$ such that $\Gamma M_{\tau^{*}}$ is Schur provide upper bounds on the minimal dwell-time between two events that ensures $\mathcal{A}$ is GAS. In the following, we establish sufficient conditions for deriving such values $\tau^{*}$.

At this point, it is interesting to emphasize that we have transformed the problem of stability analysis of the overall hybrid system in a problem of stabilization of a positive system.

Theorem 1: A sufficient condition that ensures $\mathcal{A}$ is a GAS set of the dynamics (14)-(15) $\forall i \in\{1, \ldots, N\}$ is that $t_{k+1}-t_{k} \geq \tau^{*}$ with

$$
\tau^{*}>\frac{1}{\lambda_{\varepsilon}} \max \left\{\ln \frac{\gamma_{12} c_{\varepsilon}}{1-\gamma_{11}}, \ln \frac{\gamma_{22} c_{\varepsilon}}{1-\gamma_{21}}\right\}>0 .
$$

Proof: Let us remark that

$$
\Gamma M_{\tau^{*}}=\left(\begin{array}{ll}
\gamma_{11} & \gamma_{12} c_{\varepsilon} \mathrm{e}^{-\lambda_{\varepsilon} \tau^{*}} \\
\gamma_{21} & \gamma_{22} c_{\varepsilon} \mathrm{e}^{-\lambda_{\varepsilon} \tau^{*}}
\end{array}\right) .
$$

Moreover, the positive matrix $\Gamma M_{\tau^{*}}$ is Schur if and only if there exists $z \in \mathbb{R}_{+}^{2}$, such that $\left(\Gamma M_{\tau^{*}}\right) z<z$ (see e.g. [19]). Choosing $z=\mathbb{1}_{2}$ one obtains that $\left(\Gamma M_{\tau^{*}}\right) z<z$ is equivalent with

$$
\left\{\begin{array}{l}
\gamma_{11}+\gamma_{12} c_{\varepsilon} \mathrm{e}^{-\lambda_{\varepsilon} \tau^{*}}<1 \\
\gamma_{21}+\gamma_{22} c_{\varepsilon} \mathrm{e}^{-\lambda_{\varepsilon} \tau^{*}}<1
\end{array}\right.
$$

The first inequality in (31) has the solution

$$
\tau^{*}>\frac{1}{\lambda_{\varepsilon}} \ln \frac{\gamma_{12} c_{\varepsilon}}{1-\gamma_{11}}
$$

while the second one is solved by

$$
\tau^{*}>\frac{1}{\lambda_{\varepsilon}} \ln \frac{\gamma_{22} c_{\varepsilon}}{1-\gamma_{21}} .
$$

Combining the two conditions above one obtains the result in (30). For consistency of the previous result we have to prove that either $\gamma_{11}<1$ or $\gamma_{21}<1$. Let us note that $Q\left(t_{k}\right)=U P\left(t_{k}\right) U^{\top}$ is a symmetric matrix since $P\left(t_{k}\right)$ is symmetric. Therefore $Q\left(t_{k}\right)$ is also symmetric yielding that $\left\|Q\left(t_{k}\right)\right\|$ is given by the maximum eigenvalue of $Q\left(t_{k}\right)$. This simply ensures that $\gamma_{11}<1$. Although not needed we can also prove that $\gamma_{21}<1$. Recalling the choice of $U$, we can use the Kronecker product property introduced in [20] and write $\left\|\mathcal{U}^{\top}\right\|=\left\|U^{\top} \otimes \mathbf{I}_{2}\right\|=\left\|U^{\top}\right\|\left\|\mathbf{I}_{2}\right\|=1$. Next we show that $\left\|\mathbf{I}_{2 N}-\mathcal{P}\left(t_{k}\right)\right\| \leq 1$. First of all thanks to Assumption 3 and to the stochasticity of $P\left(t_{k}\right)$ we know that $\left\|\mathbf{I}_{N}-P\left(t_{k}\right)\right\|_{1}<1$ and $\left\|\mathbf{I}_{N}-P\left(t_{k}\right)\right\|_{\infty}<1$ (it will suffice to apply the definition of the 1-norm and $\infty$-norm as the maximum of respectively columns and rows sums). Then using the following well-known matrix norm property

$$
\left\|\mathbf{I}_{N}-P\left(t_{k}\right)\right\| \leq \sqrt{\left\|\mathbf{I}_{N}-P\left(t_{k}\right)\right\|_{1}\left\|\mathbf{I}_{N}-P\left(t_{k}\right)\right\|_{\infty}}<1 .
$$

The condition $\left\|\mathbf{I}_{2 N}-\mathcal{P}\left(t_{k}\right)\right\| \leq 1$ follows once again from applying the Kronecker product property in [20].

\section{NUMERICAL EXAMPLE}

In this section we describe an illustrative application of the proposed algorithm. A set of 5 robots is considered and they are required to realize a translation invariant pentagon formation specified by the set of positions $\Pi=$ $((2,0),(3.90,1.38),(3.18,3.62),(0.82,3.62),(0.10,1.38))^{\top}$. The interaction between the agents switches randomly between the ones described by the following two stochastic matrices

$P_{1}=\left[\begin{array}{ccccc}.7 & .1 & .1 & 0 & .1 \\ .1 & .7 & .1 & .1 & 0 \\ .1 & .1 & .8 & 0 & 0 \\ 0 & .1 & 0 & .8 & .1 \\ .1 & 0 & 0 & .1 & .8\end{array}\right], P_{2}=\left[\begin{array}{ccccc}.8 & .1 & 0 & .1 & 0 \\ .1 & .7 & 0.1 & 0 & .1 \\ 0 & .1 & .7 & .1 & .1 \\ .1 & 0 & .1 & .7 & .1 \\ 0 & .1 & .1 & .1 & .7\end{array}\right]$.

A dwell-time $\tau^{*}=3.09 \mathrm{~s}$ has been used as lower bound for the intervals between an interaction and the following, where the value of $\tau^{*}$ has been evaluated using expression (30).

The initial Cartesian position of the agents have been chosen as $X_{1}(0)=(-6,-1), X_{2}(0)=(-9,-4), X_{3}(0)=(-6,3.5), X_{4}(0)=$ $(1,-2)$ and $X_{5}(0)=(-11,6)$ where the coordinates are expressed in meters; the initial heading angles are $\theta_{1}(0)=-1.5, \theta_{2}(0)=$ $-3, \theta_{3}(0)=-0.9, \theta_{4}(0)=-0.3$ and $\theta_{5}(0)=1.5$, all expressed in radians. The initial reference vectors $r_{i}(0)$ have been initialized using (12) and $X_{i}(0)$. The values of the constants $k$ and $\gamma$ for the point stabilization controller of each robot, previously described in (19), are respectively 0.8 and 1. In Fig. 2 the trajectories of the robots are shown with the sequence of reference positions $r_{i}$ updated through the consensus, emphasizing the final positions of the agents that realize the formation defined by $\Pi$ but in a different place of the $2 \mathrm{D}$ plane (i.e. what is called a translation invariant formation realization). The convergence of the 5 robots towards an agreement over the displacement $\tilde{r}_{i}(t)=r_{i}(t)-p_{i}$, with $p_{i}$ defined in $\Pi$ above, is represented in Fig. 3 and Fig. 4, respectively. The Table I collects the first 10 time intervals between consecutive references update instants. These intervals have been imposed to have random lengths within the interval $[3.09,11.09]$ i.e. all the values are lower bounded by $\tau^{*}$

TABLE I

THE LENGTH OF THE FIRST 10 TIME INTERVALS BETWEEN CONSECUTIVE RESET INSTANTS.

\begin{tabular}{|c|c||c|c|}
\hline$t_{1}-t_{0}$ & $4.25 \mathrm{~s}$ & $t_{6}-t_{5}$ & $7.51 \mathrm{~s}$ \\
\hline$t_{2}-t_{1}$ & $4.56 \mathrm{~s}$ & $t_{7}-t_{6}$ & $5.02 \mathrm{~s}$ \\
\hline$t_{3}-t_{2}$ & $5.78 \mathrm{~s}$ & $t_{8}-t_{7}$ & $4.32 \mathrm{~s}$ \\
\hline$t_{4}-t_{3}$ & $5.66 \mathrm{~s}$ & $t_{9}-t_{8}$ & $10.58 \mathrm{~s}$ \\
\hline$t_{5}-t_{4}$ & $7.48 \mathrm{~s}$ & $t_{10}-t_{9}$ & $8.92 \mathrm{~s}$ \\
\hline
\end{tabular}




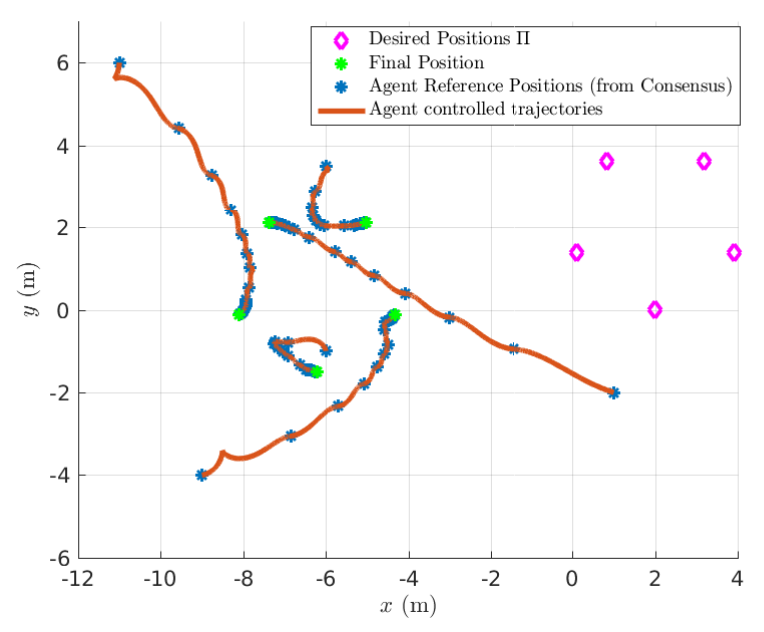

Fig. 2. Robots reaching the pentagon formation.

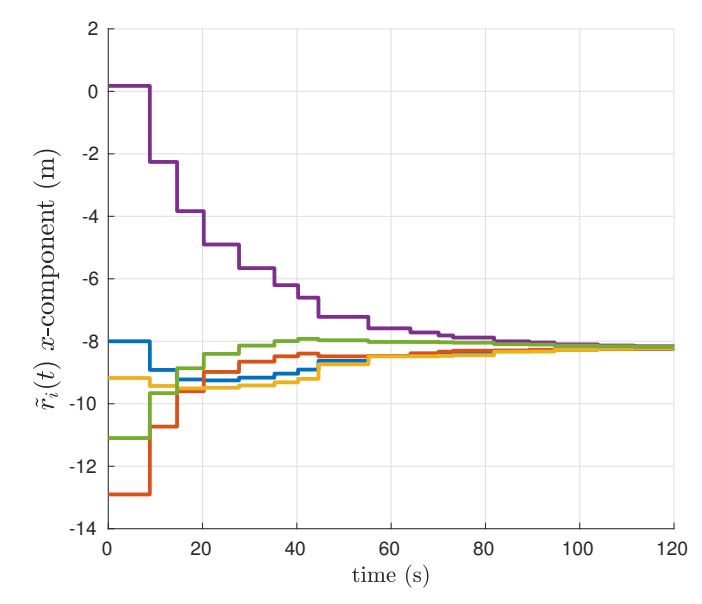

Fig. 3. Trajectories of the $x$-component of the $\tilde{r}_{i}$ for each robot.

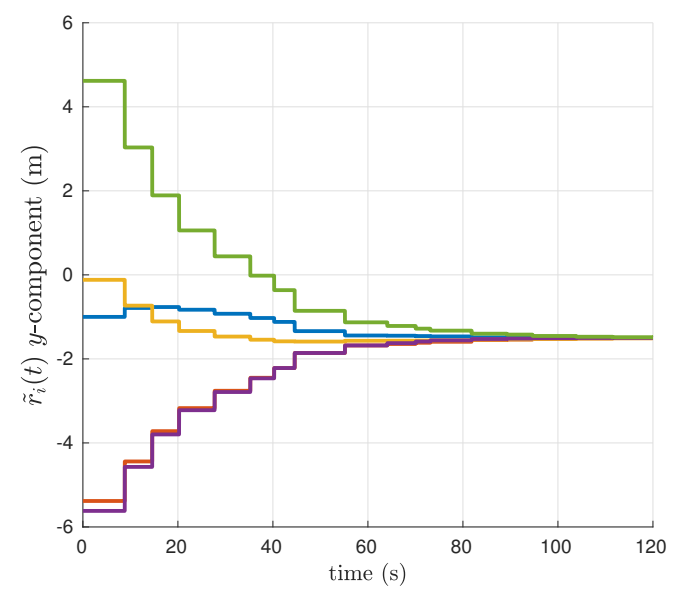

Fig. 4. Trajectories of the $y$-component of the $\tilde{r}_{i}$ for each robot.

\section{CONCLUSIONS}

We have proposed and analyzed a decentralized consensus/formation realization strategy for a fleet of non-holonomic robots. The proposed strategy requires sporadic interactions between robots. At the interaction instants the robots update their reference based on some relative inter-distance measurements. The resulting closed-loop dynamics is hybrid and our sufficient stability condition is formulated in term of a minimum dwell-time condition. A numerical example illustrates the theoretical development.

\section{REFERENCES}

[1] M. Mesbahi and M. Egerstedt, Graph theoretic methods in multiagent networks. Princeton University Press,Princeton. NY, 2010.

[2] R. Beard and V. Stepanyan, "Information consensus in distributed multiple vehicle coordinated control," in Proceedings of the 42th IEEE Conference on Decision and Control, vol. 2, 2003, pp. 2029-2034.

[3] F. Bullo, J. Cortés, and S. Martinez, Distributed Control of Robotic Networks. A Mathematical Approach to Motion Coordination Algorithms. Princeton University Press, 2009.

[4] H. Tanner, A. Jadbabaie, and G. Pappas, "Flocking in fixed and switching networks," IEEE Transactions on Automatic Control, vol. 52, no. 5 , pp. 863-867, 2007.

[5] H. Su, G. Chen, X. Wang, and Z. Lin, "Adaptive second-order consensus of networked mobile agents with nonlinear dynamics," Automatica, vol. 47, no. 2, pp. 368-375, 2011.

[6] M. Fiacchini and I.-C. Morărescu, "Convex conditions on decentralized control for graph topology preservation," IEEE Transactions on Automatic Control, vol. 59, no. 6, pp. 1640-1645, DOI.10.1109/TAC.2013.2292719 2014.

[7] Z.-P. Jiang and H. Nijmeijier, "Tracking control of mobile robots: A case study in backstepping." Automatica, vol. 33, no. 7, pp. 1393 1399, 1997.

[8] C. Samson and K. Ait-Abderrahim, "Feedback control of a nonholonomic wheeled cart in cartesian space." in in Proc. IEEE International Conference on Robotics and Automation, 1991, pp. 1136-1141.

[9] I. Kolmanovsky and N. McClamroch, "Developments in nonholonomic control problems," IEEE Control Systems Magazine, vol. 16, no. 6, pp. $20-36,1995$.

[10] R. Brockett, "Asymptotic stability and feedback stabilization." In Differential Geometric Control Theory, vol. (R.W. Brockett and R.S. Millman and H.J. Sussmann Eds), pp. 181-191, 1983.

[11] A. Astolfi, "Exponential stabilization of a wheeled mobile robot via discontinuous control," Transactions-American Society of Mechanical Engineers Journal of Dynamic Systems, Measurement, and Control, vol. 121 , pp. 121-125, 1999.

[12] C. C. de Wit and O. J. Sordalen, "Exponential stabilization of mobile robots with nonholonomic constraints," IEEE Transactions on Automatic Control, vol. 37, no. 11, pp. 1791-1797, 1992.

[13] Y. Kanayama, Y. Kimura, F. Miyazaki, and T. Noguchi, "A stable tracking control method for an autonomous mobile robot," in Proceedings of IEEE International Conference on Robotics and Automation, 1990, pp. 384-389.

[14] C.Rui and N. McClamroch, "Stabilization and asymptotic path tracking of a rolling disk," in Proceedings of the 34th IEEE Conference on Decision and Control, 1995, pp. 4294-4299.

[15] E. Panteley, E. Lefeber, A. Loria, and H. Nijmeijier, "Exponential tracking control of a mobile car using a cascaded approach," in Proceedings of IFAC Motion Control, 1998, pp. 201-206.

[16] W. Ren and R. W. Beard, "Consensus seeking in multiagent systems under dynamically changing interaction topologies," IEEE Trans. on Automatic Control, vol. 50, no. 5, pp. 655-661, 2005.

[17] A. Jadbabaie, J. Lin, and A. S. Morse, "Coordination of groups of mobile autonomous agents using nearest neighbor rules," IEEE Trans. on Automatic Control, vol. 48, no. 6, pp. 988-1001, 2003.

[18] R. Goebel, R. Sanfelice, and A. Teel, Hybrid Dynamical Systems. Princeton University Press, 2012.

[19] A. Rantzer, "Distributed control of positive systems," in Proc. of the 50th IEEE Decision and Control and European Control Conference, 2011, pp. 6608-6611.

[20] P. Lancaster and H. Farahat, "Norms on direct sums and tensor products," mathematics of computation, vol. 26, no. 118, pp. 401-414, 1972. 